\title{
Genetic Algorithm based Input Selection for a Neural Network Function Approximator with Applications to SSME Health Monitoring
}

\author{
Charles C. Peck and Atam P. Dhawan \\ Dept. of Electrical and Computer Engineering \\ University of Cincinnati Cincinnati, OH 45221
}

\author{
Claudia M. Meyer \\ Sverdrup Technology, Inc. \\ NASA Lewis Research Center Group \\ Brook Park, OH 44142
}

\begin{abstract}
A genetic algorithm is used to select the inputs to a neural network function approximator. In the applicatiou considered, modeling critical parameters of the Space Shuttle Main Engine (SSME), the functional relationship between measured parameters is unknown and complex. Furthermore, the number of possible input parameters is quite large. Many approaches have been used for input selection, but they are either subjective or do not consider the complex multivariate relationships between parameters. Due the optimization and space searching capabities of genetic algorithms they were employed in this paper to systematize the input selection process. The results suggest that the genetic algorithm can generate parameter lists of high quality without the explicit use of problem domain knowledge. Suggestions for improving the performance of the input selection process are also provided.
\end{abstract}

\section{INTRODUC'TION}

There is considerable interest. within the space industry in improving the fault detection and isolation capabilities of rocket engine condition monitoring and post-test diagnostic processing systems. This requires developing accurate models of engine parameters based on other parameters measured from the engine. Developing accurate models is particularly difficult due to the highly complex, non-linear nature of rocket engines, the limited suite of measured parameters, and the large variability of behavior among engines of the same design.

It has been shown that neural networks with one hidden layer can uniformly approximate any continuous function $[1,2,3]$. Furthermore, neural net,works are well-suited for problems in which the exact relationships between inputs and outputs are complex or unkuown $[4,1]$. These conclusions may be applied to dynamical systems if the system state is sufficiently represented in the inputs of the neural network. For these reasons, feedforward neural networks have been used to inodel critical parameters of the Space Shuttle Main Engine (SSME) during the start-nip trausient and they have been shown to be quite effective [4].

A trask that is critical to the success of neural network modeling of complex, dynamical systems such as the SSME is the choice of the imput parameters. There are several constraints that complicate this task. First, while the instrumentation of the SSME is extensive, it is not complete. Therefore, it is unlikely that it will be possible to completely describe iny subsystem input, or output. Second, as was discussed above, it is necessary to provide enough state information to model the desired parameter. Finally, it is not practical to use a large number of inputs for a number of reasons. First, a time window of each input parameter is typically used in order to provide time dependent information. The size of the window multiplies the number inputs to the network. For example if 10 parameters are chosen as the network inputs and a time window of the past ten values is used, then the effective number of inputs to the network is 100 . Another reason that the input set should be restricted is that large networks are difficult to train. Finally, the input. set should 
be small if the system is to be used for real-time modeling

A number of ad hoc approaches have been proposed or used for input selection. These include the use of characteristic equations, engine schematic analysis, correlations between candidate input parameters and the modeled parameter, and expert advice. These methods are highly subjective or they do not adequately measure the multivariate dependencies present in the system. For these reasons, a systematic approach for input selection is desired.

The choice of inputs may be modeled as an optimization problem where the space of possible solutions is quite large. In fact, roughly 500 sensors are used for monitoring during test firings of the SSME. This represents approximately $2^{5010}$ (listinct input sets. Since an exhaustive search is clearly not possible, an alternative search method is required.

Genetic algorithms are well suited for searching in a large parameter space $[5,6]$. Through the use of seeding (the process of providing an initial set, of possible solutions), genetic algorithms search from a set of solutions or starting points, rather than a siugle starting point. Genetic algorithms are not derivative based, thus they can search spaces where methods such as conjugate descent fail. They work with both discrete and continuous parameters, and explore and exploit, the parameter space [7]. Furthermore, through the use of elitism (a variant method in which the best solution of a genteration is promoted unaltered to the next generation), a genetic algorithm can be guaranteed to perform at least as well the methods used to seed or initialize it. For these reasons, a genetic algorithm was used in this paper to select the inputs to a neural network used for SSME parameter modeling during the start-up transient.

This paper will first present the design issues and methodology applied to the selection of SSME input parameters. A presentation and discussion of results will follow. The paper will conclude with the conclusions and ideas for future work.

\section{DESIGN ISSUES AND METHODOLOGY}

The design issues range from those applicable to all genetic algorithms and multi-layered perceptron neural networks to those specific to this particular problem of SSME parameter approximation.

There are two fundamental design requirements for applying genetic algorithms: encoding candidate solutions onto binary strings, and developing a fitness function. In this case, encoding candidate solutions onto binary strings is trivial since a single bit is sufficient to indicate whether a particular parameter is to be included in the network input set. Accordingly, the string, or chromosome, has one bit for every candidate engine parameter. To reduce the size of the search space, redundant sensor measurements were eliminated and those parameters believed to be nearly independent of the modeled parameter were not included in the candidate parameter set. This reduced the size of the candiclate parameter set to 49 parameters. Before discussing the developinent of the fitness function, it should be noted that in the genetic algorithm used, the smaller the fitness function value, the better the evaluated solution is considered to be.

The choice of a fitness function is somewhat more complicated than the string encoding. Recall that the primary function of the genetic algorithm is to produce input sets that enable neural network function approximators to accurately learn and generalize the relationships between the modeled parameter and the input parameters. Oue way to do this is to make the fitness function proportional to the neural network training error. Adding the input set. size constraint to the fitness function could be done simply by multiplying the training error by the number of parameters selected. This results in a very strong constraint, however. The strength of the size constraint can be controlled by adding a constant to the number of parameters selected. A small offset created in this manner yields a strong size constraint, whereas a large offset yields a weak one. The fitness function may be further tweaked by squaring the size constraint term. This increases the strength of the constraint is the number of parameters increases.

The additional need to minimize the number of inputs to the network and the disparity in the size between henristically and randomly selected seeding sets are primarily responsible for the added complexity of the fitness function. The heuristically selected seeding sets consist of approximately 10 parameters, while the randomly selected seeding sit.s consist of approximately 25 parameters. If the two seeding sets were approximately the same size, an offset. could be chosen that would yield the desired input set. size at the 
end of the evolution process. This size disparity, however. results in either a strongly biased choice of input parameters or it results in input, set.s that are too large. To see this, consider the use of an offset sufficient to reduce the randomly selected seeding sets to a target. size of 8 parameters. Due to the size disparity, the heuristically selected seeding sets would have considerably lower fitness function values and would thus dominate in successive generations. Conversely, the use of an offset that does not significantly favor the heuristically selected seedings may not. significantly reduce the size of the parameter lists.

For the work presented in this paper, generation dependent, offsets were used to avoid biasing the results while ensuring satisfaction of the size constraint. Initially, the offset was set very high to allow the candidate solutions to compete primarily on the basis of the training error. As the genetic algorithm proceeded, the size constraint was made progressively stronger. By the end of the genetic algorithm the offset was small, yielding a strong bias for shorter lists. This change of offset. with respect to the generation will be referred to as an offset progression. Two offset progressions were used: one yielding a generally weak size constraint, and another yielding a generally strong size constraint. The offset progression yielding the weaker size constraint ranged from 71 initially to 14 over 20 generations. The other ranged from 45 initially to 7 over 20 generations. The resulting fitness functions are shown in Equations 1 and 2, respectively:

$$
\begin{aligned}
& f=\frac{(c+71-3 G)^{2}}{(71-3 G)^{2}} \times \text { Training Error, } \\
& f=\frac{(c+45-2 G)^{2}}{(45-2 G)^{2}} \times \text { Training Error, }
\end{aligned}
$$

where $f$ is the fitness function value, $c$ is the number of parameters in the candidate input, list, and $G$, which ranges from 0 to 19 , is the generation number.

To ensure robustness and resistance to domination by "Super Individuals" (i.e., non-optimal solutions that are significantly more fit than other solutions early in the evolution process), the evolutionary process was designed to run in two stages. In the first stage, three populations were independently evolved. These populations were used to seed a second evolutionary stage. In the first stage, fitness functions with weaker size constraints were used. This favors lower training error. In the second stage, the fitness function with the stronger size constraint was used.

To further increase diversity within the "rene pool," the fitness functions in two of the first stage genetic algorithms were varied to favor either early or late convergence of the neural network training error. The method used to implenent these biases exploits the observation that the training error consistently remained on a high plateau before falling precipitonsly, as shown in Figure 1. Since oscillations and unusual patterns in the training error were not observed, integration of the area bounded by the error curve and a bounding rectangle could be performed. To favor early convergence, the fitness function in Equation 1 was multiplied by the area of integration normalized by the area of the bounding box. If $A, B$, and $C$ denote the normalized areas of their corresponding regions in Figure 1, the shape dependent fitness term is $A$ for the early training error curve and $A+B$ for the late training error curve. To favor late convergence, the normalized area of integration is first subtracted from 1 before multiplying Equation 1. This corresponds to a shape dependent. fitness term of $B+C$ for the early training error curve and $C$ for the late training error curve.

As described above, the fitness function evaluation involves creating a nenral network, training it, and evaluating its performance. This is computationally expensive and time consuming. To limit the cost of performing this operation, the QuickProp learning algorithm was nsed [8]. Furthermore, the network was trained only as far as necessary to distinguish it. from other networks with different input configurations. It was determined empirically that 100 epochs is sufficient. According to the analysis provided in [8], this should be comparable to 1000 epochs of training with standard backpropagation. Finally, the neural networks were presented with a time window of 5 past values instead of the 10 past values used in [4].

Another important design consicleration is that the training error of a network is a noisy fitness evaluation function. The weight initialization can have a significant, effect on the performance of a network. Thus, to avoid biasing the fitness of a particular candiclate set of inputs as either too good or too bad, the fitness of each candidate input set, was evaluated every generation in which it was present.

III. RESULTS AND DISCUSSION 


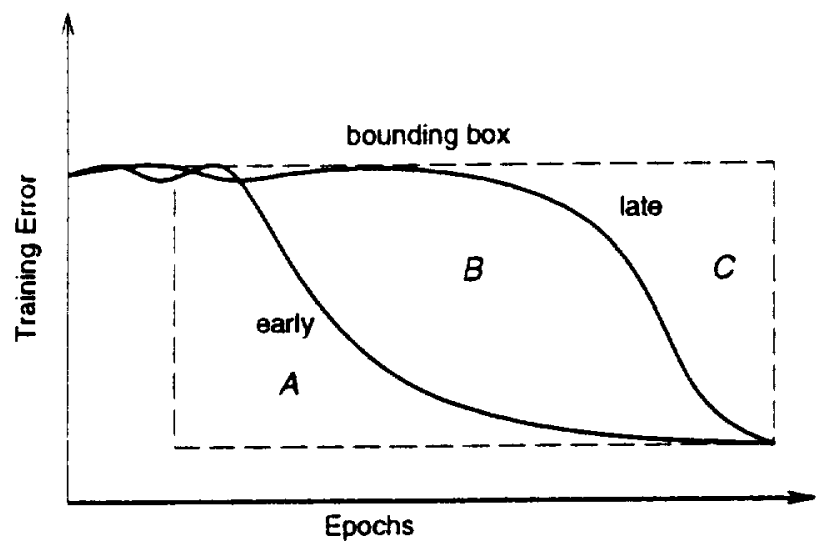

Figure 1: Early versus Late Training Error Convergence

Table 1: Parameter Lists

\begin{tabular}{|c|c|c|}
\hline $\begin{array}{c}\text { Parameter } \\
\text { List }\end{array}$ & $\begin{array}{c}\text { Number } \\
\text { of PIDs }\end{array}$ & Parameters \\
\hline \hline GA-1 & 6 & 21582097349511050 \\
\hline GA-2 & 7 & 21582093277349511058 \\
\hline GA-3 & 8 & 2152582093277349511050 \\
\hline REF & 9 & 40425923148012051212 O/Cs OPBs \\
\hline
\end{tabular}

The fundamental output of the system described above consists of candidate parameter lists. The three parameter lists with the best. fitness values are presented in Table 1. These three lists are labeled GA-1GA-3. An additional list, labeled REF, is also presented for the purpose of comparison. This "reference" list has been modified from the one presented in [4] to exclucle autoregressive information.

The parameter that was modeled is the SSME's High Pressure Oxidizer Turbine (HPOT) discharge temperature, which has a Parameter IDentification number (PID) of 233. Descriptions of this PID and the others included in the four lists described above are provided in Table 2.

To evaluate the performance of the parameter lists produced by the genetic algorithms, feedforward neural networks were fully trained using these lists and the reference list. The resulting networks were then used to approximate PID 2333 using measured parameters from 12 actual SSME test firings. Four of the test firings were used for training the networks and eight, were used to validate the resulting models. The results, as represented by the mean squared error (MSE), the normalized MSE, and the maximum percent error, are shown in Tables $3,4,5$, and 6 . A summary of these results is presented in Tables 7 and 8 . The results are divided into two groups: one presenting the aggregate performance of the networks on the training data (Table 7), and the other presenting the aggregate performance of the networks on the validation data (Table 8).

It is clear from the results that the parameter list. GA-1 has the worst error performance of the four lists. This is compensated by the fact that this is the shortest parameter list. Even though the error performance of this parameter list is the worst. it is still close to the performance of the other lists, including the reference list.

The parameter lists GA-2 and GA-3 outperformed the reference list on the training data and performed only slightly worse than the reference list on the validation clata. Due to the large standard deviations of validation data error, the differences in the error means cannot. be considered statistically significant. 
Table 2: Parameter Descriptions

\begin{tabular}{|l|l|}
\hline PID & Description \\
\hline \hline 21 & Main Combustion Chamber Oxidizer Injection Temperature \\
\hline 40 & Oxidizer Preburner Oxidizer Valve Actuator Position \\
\hline 42 & Fuel Preburner Oxidizer Valve Actuator Position \\
\hline 52 & High Pressure Fuel Pump Discharge Pressure \\
\hline 58 & Fuel Preburner Chamber Pressure \\
\hline 59 & Preburner Boost Pump Discharge Pressure \\
\hline 209 & High Pressure Oxidizer Pump Inlet. Pressure \\
\hline 231 & High Pressure Fuel Turbine Discharge Temperature \\
\hline $233 \dagger$ & High Pressure Oxidizer Turbine Discharge Temperature \\
\hline 327 & High Pressure Oxidizer Pump BalCav \\
\hline 480 & Oxidizer Preburner Chamber Pressure \\
\hline 734 & Low Pressure Oxidizer Pump Speed \\
\hline 951 & High Pressure Oxidizer Pump Pressure SL DR \\
\hline 1050 & Oxidizer Tank Discharge Temperature \\
\hline 1058 & Engine Oxidizer Inlet Temperature \\
\hline 1205 & FAC. Fuel Flow \\
\hline 1212 & FAC. Oxidizer Flow \\
\hline O/Cs & Dummy Parameter indicating Open/Closed Loop Operation \\
\hline OPBs & Dummy Parameter indicating Oxidizer Preburner Prime Time \\
\hline$\dagger$ the modeled parameter \\
\hline
\end{tabular}

Table 3: Error Statistics from Parameter List GA-1

\begin{tabular}{|c|c|c|c|c|}
\hline $\begin{array}{c}\text { Test } \\
\text { Firing }\end{array}$ & $\begin{array}{c}\text { Training/ } \\
\text { Valiclation }\end{array}$ & MSE & NMSE & $\begin{array}{c}\text { Max. } \\
\text { \% Error }\end{array}$ \\
\hline \hline B1046 & $\mathrm{T}$ & 3.787033 & 0.000322 & 2.2330 \\
\hline B1060 & $\mathrm{T}$ & 14.743364 & 0.001223 & 4.8150 \\
\hline B1061 & $\mathrm{V}$ & 20.168583 & 0.001657 & 10.4348 \\
\hline B1062 & $\mathrm{V}$ & 34.029559 & 0.002832 & 9.6225 \\
\hline B1063 & $\mathrm{V}$ & 39.671779 & 0.0033301 & 6.9063 \\
\hline B1066 & $\mathrm{V}$ & 30.608499 & 0.002532 & 7.5330 \\
\hline B1067 & $\mathrm{V}$ & 42.103255 & 0.003498 & 9.2189 \\
\hline B1070 & $\mathrm{T}$ & 11.699498 & 0.000945 & 3.1922 \\
\hline B1071 & $\mathrm{V}$ & 63.607371 & 0.005154 & 20.8187 \\
\hline B1072 & $\mathrm{V}$ & 23.816642 & 0.001898 & 8.3420 \\
\hline B1075 & $\mathrm{V}$ & 20.268258 & 0.0016699 & 10.0018 \\
\hline B1077 & $\mathrm{T}$ & 12.931541 & 0.001045 & 5.3681 \\
\hline
\end{tabular}


Table 4: Error Statistics from Parameter List GA-2

\begin{tabular}{|c|c|c|c|c|}
\hline $\begin{array}{c}\text { Test } \\
\text { Firing }\end{array}$ & $\begin{array}{c}\text { Training/ } \\
\text { Validation }\end{array}$ & MSE & NMSE & $\begin{array}{c}\text { Max. } \\
\text { \% Error }\end{array}$ \\
\hline \hline B1046 & $\mathrm{T}$ & 3.341027 & 0.000284 & 2.0253 \\
\hline B1060 & $\mathrm{T}$ & 6.059692 & 0.000503 & 3.1339 \\
\hline B1061 & $\mathrm{V}$ & 19.080619 & 0.001568 & 5.9461 \\
\hline B1062 & $\mathrm{V}$ & 37.601837 & 0.003129 & 9.9597 \\
\hline B1063 & $\mathrm{V}$ & 35.212338 & 0.002930 & 6.6999 \\
\hline B1066 & $\mathrm{V}$ & 33.799122 & 0.002796 & 7.3425 \\
\hline B1067 & $\mathrm{V}$ & 36.724494 & 0.003051 & 7.9440 \\
\hline B1070 & $\mathrm{T}$ & 10.692421 & 0.000864 & 3.6021 \\
\hline $\mathrm{B} 1071$ & $\mathrm{~V}$ & 48.479267 & 0.003929 & 15.9965 \\
\hline B1072 & $\mathrm{V}$ & 17.781945 & 0.001417 & 5.1814 \\
\hline B1075 & $\mathrm{V}$ & 35.017457 & 0.002884 & 11.4959 \\
\hline $\mathrm{B} 1077$ & $\mathrm{~T}$ & 7.973934 & 0.000644 & 2.6040 \\
\hline
\end{tabular}

Table 5: Error Statistics from Parameter List GA-3

\begin{tabular}{|c|c|c|c|c|}
\hline $\begin{array}{c}\text { Test } \\
\text { Firing }\end{array}$ & $\begin{array}{c}\text { Training/ } \\
\text { Validation }\end{array}$ & MSE & NMSE & $\begin{array}{c}\text { Max. } \\
\text { \% Error }\end{array}$ \\
\hline \hline B1046 & $\mathrm{T}$ & 4.015642 & 0.000341 & 1.7042 \\
\hline B1060 & $\mathrm{T}$ & 6.114787 & 0.000507 & 2.5343 \\
\hline B1061 & $\mathrm{V}$ & 20.477665 & 0.001682 & 6.2484 \\
\hline $\mathrm{B} 1062$ & $\mathrm{~V}$ & 40.542411 & 0.003374 & 10.5837 \\
\hline $\mathrm{B} 1063$ & $\mathrm{~V}$ & 38.320758 & 0.003188 & 7.1349 \\
\hline $\mathrm{B} 1066$ & $\mathrm{~V}$ & 38.782970 & 0.003208 & 8.8021 \\
\hline $\mathrm{B} 1067$ & $\mathrm{~V}$ & 39.245907 & 0.003261 & 8.4381 \\
\hline $\mathrm{B} 1070$ & $\mathrm{~T}$ & 10.516996 & 0.000850 & 3.2462 \\
\hline $\mathrm{B} 1071$ & $\mathrm{~V}$ & 53.008396 & 0.004296 & 18.9413 \\
\hline $\mathrm{B} 1072$ & $\mathrm{~V}$ & 17.990471 & 0.001434 & 4.7040 \\
\hline $\mathrm{B} 1075$ & $\mathrm{~V}$ & 33.172788 & 0.002732 & 12.0369 \\
\hline $\mathrm{B} 1077$ & $\mathrm{~T}$ & 6.889614 & 0.000557 & 2.3684 \\
\hline
\end{tabular}


Table 6: Error Statistics from Parameter List REF

\begin{tabular}{|c|c|c|c|c|}
\hline $\begin{array}{c}\text { Test } \\
\text { Firing }\end{array}$ & $\begin{array}{c}\text { Training/ } \\
\text { Validation }\end{array}$ & MSE & NMSE & $\begin{array}{c}\text { Max. } \\
\text { \% Error }\end{array}$ \\
\hline \hline B1046 & T & 6.652181 & 0.000565 & 3.8462 \\
\hline B1060 & T & 7.375382 & 0.000612 & 3.0370 \\
\hline B1061 & V & 22.370471 & 0.001838 & 4.8509 \\
\hline B1062 & V & 23.747774 & 0.001976 & 7.2832 \\
\hline B1063 & V & 28.076726 & 0.002336 & 7.9618 \\
\hline B1066 & V & 16.538060 & 0.001368 & 7.6115 \\
\hline B1067 & V & 20.482848 & 0.001702 & 6.6011 \\
\hline B1070 & T & 6.588053 & 0.0005332 & 3.8668 \\
\hline B1071 & V & 50.654580 & 0.004105 & 11.0878 \\
\hline B1072 & V & 42.897089 & 0.003419 & 6.7544 \\
\hline B1075 & V & 25.213499 & 0.002077 & 9.4449 \\
\hline B1077 & T & 7.809484 & 0.000631 & 4.5456 \\
\hline
\end{tabular}

Table 7: Summary of Parameter List Performance on Training Data

\begin{tabular}{|c|c|c|c|c|c|c|}
\hline \multirow{2}{*}{$\begin{array}{c}\text { Parm. } \\
\text { List }\end{array}$} & \multicolumn{2}{|c|}{ MSE } & \multicolumn{2}{c|}{ NMSE } & \multicolumn{2}{c|}{ Max. } \\
\cline { 2 - 7 } & $\mu$ & $\sigma$ & $\mu$ & $\sigma$ & $\mu$ & $\sigma$ \\
\hline \hline GA-1 & 10.7903359 & 4.8333357 & 0.000884 & 0.000392 & 3.902086 & 1.445958 \\
\hline GA-2 & 7.016768 & 3.101272 & 0.000574 & 0.000244 & 2.841333 & 0.679829 \\
\hline GA-3 & 6.884260 & 2.709112 & 0.000564 & 0.000212 & 2.463281 & 0.633292 \\
\hline REF & 7.106275 & 0.589258 & 0.000585 & 0.000045 & 3.823920 & 0.617108 \\
\hline
\end{tabular}

Table 8: Summary of Parameter List Performance on Validation Data

\begin{tabular}{|c|c|c|c|c|c|c|}
\hline \multirow{2}{*}{$\begin{array}{c}\text { Parm } \\
\text { List }\end{array}$} & \multicolumn{2}{|c|}{ MSE } & \multicolumn{2}{c|}{ NMSE } & \multicolumn{2}{c|}{ Max. } \\
\cline { 2 - 7 } & $\mu$ & $\sigma$ & $\mu$ & $\sigma$ & $\mu$ & $\sigma$ \\
\hline \hline GA-1 & 34.284241 & 14.485731 & 0.002818 & 0.001180 & 10.359750 & 4.397353 \\
\hline GA-2 & 32.962132 & 10.068242 & 0.002713 & 0.000832 & 8.820735 & 3.563816 \\
\hline GA-3 & 35.1926733 & 11.3493328 & 0.002897 & 0.000937 & 9.611175 & 4.430940 \\
\hline REF & 28.7476311 & 11.808645 & 0.0023353 & 0.000932 & 7.699442 & 1.889502 \\
\hline
\end{tabular}


It should be noted that the heuristically chosen parameter lists that were used to seed the genetic algorithms were outperformed early in the process by genetic algorithm generated parameter lists. While the behavior and results of the genetic algorithm were certainly affected by the heuristically chosen parameter sets, the guidance provided by these sets did not appear to be strong.

\section{CONCLUSIONS AND FUTURE WORK}

The results indicate that the error performance of the genetic alyorithm generated parameter lists was roughly the same as that of the reference list. Furthermore, in all cases, the genetic algorithm generated parameter lists were smaller than the reference list. Thus, the genetic algorithm was able to systematically generate parameter lists that performed well without the explicit use of problem domain knowledge.

Many improvements for the input, selection process have been envisaged. One may, for example, modify the fitness evaluation function to be dependent on the error on a validation set instead on the training. This would favor parameter lists that yield networks with superior generalizing capabilities instead of lists that yield networks capable of rapid learning. As an extension, the fitness function could be made a function of the training error, the validation error, and the generation. In this manner, learning capability could be favored early in evolution and generalization could be favored later.

As demonstrated by the GA-1 list, smaller size can be overemphasized compared to the error performance. Instead of favoring a parameter list of the smallest size, a list of a particular size conld be favored. This would favor the inclusion of sufficient information while discouraging the use of parameters that do not significantly improve the error performance. For this particular application, a size of 10 would be reasonable.

\section{ACKNOWLEDGMENTS}

The public domain genetic algorithm GENESIS Version 5.0, written by John J. Grefenstette, was used for the work described in this paper. Furthermore, the fitness evaluation function is a highly modified and optimized derivative of Terry Regier's implementation of the QuickProp training algorithm.

\section{REFERENCES}

[1] S. Chen, S. A. Billings, and P. M. Grant. Non-linear systems identification using neural networks. Research Report 370, University of Edinburgh, Mayfield Road, Edinburgh, Scotland, August 1989.

[2] G. Cybenko. Approximation by superpositions of a sigmoidal function. Mathematics of Control, Signals, and Systems, 2:303-314, 1989.

[3] K. Funahashi. On the approximate realization of continuous mappings by neural networks. Neural Networks, 2:183-192, 1989.

[4] Claudia M. Meyer and William A. Maul. The application of neural networks to the ssme startup transient. Number 2530 in 91 . AIAA, July 1991.

[5] David E. Goldberg. Genctic Algorithms in Search, Optimization, and Machine Learning. Addison-Wesley Publishing Company, Inc., Reading, Massachuset.ts, 1989.

[6] Lawrence Davis. Haudbook of (ienetic Algorithms. Van Nostrand Reinhold, New York, 1991.

[7] David J. Powell, Michael M. Skolnick, and Siu Shing Tong. Handbook of Gentic Alyorthms, chapter 20, pages 312-331. Van Nostrand Reinhold, New York, 1991.

[8] Scott E. Fahlman. Faster-learning variations on back-propagation: An empirical study. In D. Touretzky, G. Hinton, and T. Sejnowski, editors, Proccedings of the 1988 Connectionist Models Summer School, pages 38-51, San Mateo, CA, hune 1988. Carnegie Mellon University, Morgan Kaufmann Publishers. 